ORIGINAL ARTICLE

\title{
Diabetes services in the UK: fourth national survey; are we meeting NSF standards and NICE guidelines?
}

\author{
J A Edge, P G F Swift, W Anderson, B Turner, on behalf of the Youth and Family \\ Advisory Committee of Diabetes UK
}

See end of article for authors' affiliations

Correspondence to: Dr J A Edge, Consultant in Paediatric Diabetes and Endocrinology, John Radcliffe Hospital, Oxford OX3 9DU, UK; julie. edge@paediatrics.ox.ac. uk

Accepted 24 May 2005 Published Online First 7 June 2005
Background: Previous surveys of children's diabetes service provision in the UK have shown gradual improvements but continuing deficiencies.

Aim: To determine whether further improvements in services have occurred.

Methods: A questionnaire was mailed to all paediatricians in the UK identified as providing care for children and adolescents with diabetes. Responses were compared with results of three previous surveys, and with recommendations in the Diabetes NSF and the NICE type 1 diabetes guidelines.

Results: Replies were received from 187 consultant paediatricians in 169 centres looking after children; $89 \%$ expressed a special interest in diabetes, $98 \%$ saw children in a designated diabetic clinic, and $95 \%$ clinics now have more than 40 patients. In $98 \%$ of the clinics there was a specialist nurse $182 \%$ now children's trained), but $61 \%$ clinics had a nurse:patient ratio $<1: 100 ; 39 \%$ of clinics did not have a paediatric dietician and in $78 \%$ there was no access to psychology/psychiatry services in clinics. Glycated haemoglobin was measured routinely at clinics in $86 \%$, annual screening for retinopathy performed in $80 \%$, and microalbuminuria in $83 \%$. All centres now have local protocols for ketoacidosis, but not for children undergoing surgery $(90 \%)$ or severe hypoglycaemia (74\%). Mean clinic $\mathrm{HbAlc}$ levels were significantly lower in the clinics run by specialists (8.9\%) than generalists $(9.4 \%)$. There have been incremental improvements over the last 14 years since the surveys began, but only two clinics met all the 10 previously published recommendations on standards of care.

Conclusions: The survey shows continuing improvements in organisational structure of services for children with diabetes but serious deficiencies remain. Publication and dissemination of the results of the previous surveys may have been associated with these improvements and similar recurrent service review may be applicable to services for other chronic childhood conditions.
$\mathrm{P}$ revious surveys of services provided for children and adolescents with type 1 diabetes showed deficiencies when compared with national recommendations. ${ }^{1-3}$ Since 1998, the Diabetes National Service Framework (NSF) has set targets and standards ${ }^{4}$ and the National Institute of Clinical Excellence (NICE) has published guidelines for the management of type 1 diabetes. ${ }^{5}$

The Youth and Families Advisory Committee of Diabetes UK agreed to carry out a further survey to measure current provision of services against these guidelines.

\section{METHODS}

The National Diabetes Audit project run jointly by Diabetes UK and the British Society of Paediatric Endocrinology and Diabetes identified 263 consultants in 216 hospitals caring for children and young people with diabetes in the UK. A postal questionnaire was sent to all these consultants in 2002 seeking information about staffing levels, personnel, clinic size and type, facilities, and patterns of care. Results were compared with the three previous surveys, ${ }^{1-3}$ to 10 key recommendations from "Principles of Good Practice for the Care of Young People with Diabetes" ${ }^{\prime 6}$ and to recommendations from the Diabetes NSF ${ }^{4}$ and NICE guidelines. ${ }^{5}$

\section{RESULTS}

A total of 187 (71\%) completed questionnaires were returned from clinics in 169 (78\%) hospitals identified. A total of 187 consultants reported that they care for around 18500 children and young people with diabetes (15 400 under the age of 16 years).

Key results of the survey and comparisons with results from 1988, 1994, and 1998 are summarised in table 1. Results are generally described by hospital, except for those questions relating specifically to individual consultants.

\section{Specialist interest of consultants}

- Recommendation: Children with diabetes should be under the care of consultants with a special interest and training in diabetes. ${ }^{\circ}$

Twenty one consultants $(11 \%)$ indicated that they were paediatric endocrinologists/diabetologists; 90 (48\%) considered themselves to have a special interest in diabetes, and 54 $(29 \%)$ a special interest in diabetes/endocrinology. One was an adult diabetologist. The remaining $21(11 \%)$ regarded themselves as general paediatricians.

\section{Types of clinic}

- Recommendation: Children with diabetes should be seen in a designated paediatric diabetes clinic. ${ }^{6}$

Abbreviations: DSN, diabetes specialist nurse; NICE, National Institute of Clinical Excellence; NSF, National Service Framework; WTE, whole time equivalent 
Table 1 Comparison of results of four national surveys

\begin{tabular}{lllll}
\hline & 1988 & 1994 & 1998 & 2002 \\
\hline Total number of consultant respondents & 360 & 325 & 244 & 187 \\
Response rate & $86 \%$ & $94 \%$ & $81 \%$ & $78 \%$ \\
Consultant with a special interest in diabetes & $32 \%$ & $48 \%$ & $78 \%$ & $89 \%^{*}$ \\
Consultant caring for more than 40 young people & $23 \%$ & $45 \%$ & $74 \%$ & $94 \%^{*}$ \\
Designated paediatric diabetes clinic & $63 \%$ & $88 \%$ & $91 \%$ & $98 \%$ \\
Diabetes specialist nurse in clinic & $61 \%$ & $87 \%$ & $93 \%$ & $98 \% \dagger$ \\
Paediatric dietician in clinic & $37 \%$ & $57 \%$ & $65 \%$ & $61 \%$ \\
Psychologist/psychiatrist in clinic & $9 \%$ & $22 \%$ & $22 \%$ & $22 \% \ddagger$ \\
Glycated haemoglobin at each visit & $91 \%$ & $90 \%$ & $88 \%$ & $86 \%$ \\
\hline *Significant difference 1998-2002, p<0.001. & & & & \\
†Significant difference 1998-2002, p<0.025. & & & \\
†Additional 28\% with some form of "counsellor" in clinic. & & & \\
\hline
\end{tabular}

One hundred and sixty four clinics (98\%) are now designated paediatric diabetes clinics. Diabetic children are seen in general paediatric clinics in only $2 \%$ of hospitals.

- NICE Guideline 7.2: Paediatric diabetes care teams should organise age banded clinics for adolescents and young adults jointly with their adult colleagues. ${ }^{5}$

Seventy one per cent of hospitals $(n=119)$ have age stratified clinics; of these $63 \%$ have an adolescent clinic. Forty eight per cent of the centres have joint clinics with adult colleagues.

\section{Clinic size}

- Recommendation: In districts with fewer than 70 children with diabetes under the age of 16 years, all should be under the care of a single designated consultant paediatrician. ${ }^{6}$

Seventy five clinics (45\%) cared for more than 100 children and adolescents with diabetes; 82 (49\%) care for 40-100, with the remaining nine clinics $(6 \%)$ caring for fewer than 40 young people with diabetes. Thirty nine clinics $(23 \%)$ had fewer than 70 patients. Twenty nine of these were managed by paediatricians with a special interest in diabetes, and nine were general paediatricians. In only one of these was there more than one consultant. Of the clinics run by general paediatricians, two had more than 100 patients. These figures represent a significant shift towards consultant specialisation and being responsible for larger caseloads since 1994 and 1998 (table 1; $\chi^{2}=121.6, \mathrm{p}<0.001$ ).

\section{Diabetes specialist nurses}

- Recommendation: Clinics should be supported and attended by a paediatric DSN with children's and diabetes training and with a maximum caseload of 70-100 children per nurse (full time equivalent). ${ }^{7}$

Diabetes specialist nurses (DSNs) attended 165 clinics (98\%). In $53 \%$ of these the nurses worked solely in paediatric diabetes; the remainder either covered another paediatric sub-specialty $(19 \%)$ or also cared for adults with diabetes $(28 \%)$. Eighty two per cent of DSNs were trained in children's nursing, $9 \%$ were not able to spend time in the community.

In the $124(73 \%)$ clinics which responded to the question about nurses' working hours, an average of 1.83 DSNs were working 1.05 whole time equivalents (WTE). The average caseload per WTE DSN was 147 (range 12-575). There was no difference in the caseload of the DSNs in clinics run by general paediatricians (mean 129), paediatricians with an interest in diabetes (140), an interest in diabetes and endocrinology (159), and paediatric endocrinologists (130). Thirty nine per cent of clinics had a specialist nurse. WTE:patient ratio $\leqslant 100$.

\section{Dietetic support}

- Recommendation: Paediatric diabetes clinics should have regular attendance of a paediatric dietician with diabetes expertise. $^{6}$

Dieticians were reported to attend regularly at $87 \%$ of clinics with 114 (61\% of total) being a paediatric dietician.

The average number of paediatric dieticians WTE per clinic was 0.29 (range 0.04-1.5) with average WTE:patient ratio 830 (range 24-3750).

\section{Psychologist/counsellor services}

- Recommendation: The clinic has easy access to mental health professionals ${ }^{5}$ or specialist services in child psychology/psychiatry. ${ }^{6}$

Twenty two per cent of respondents reported that either a psychologist $(20 \%)$ or psychiatrist $(2 \%)$ regularly attended the children's diabetes clinic. Forty six clinics $(28 \%)$ had access to another form of "counsellor" (nurse therapist in 20; counsellor in 11; and "other" in 12). Eighty seven per cent of clinics could refer to a psychologist, and $90 \%$ to a psychiatrist; waiting times for these specialists were reported to range from 1 week to 18 months.

\section{Social worker availability}

Thirty clinics (18\%) reported access to social work support and $16(53 \%)$ of these reported that the social worker could carry out home visits.

\section{Chiropody services}

- Recommendation: Children's diabetes clinics should have easy access to chiropody services. ${ }^{6}$

Fifty nine clinics (36\%) reported access to chiropody services, mainly hospital chiropody. In 1998, 56\% clinics reported access to chiropody.

\section{Measurement of glycaemic control}

- NICE Guideline 4.5: Current HbAlc measurements should be made available for use and/or discussion in outpatient clinics. ${ }^{5}$

Glycated haemoglobin (HbAlc) is measured at every clinic visit in $86 \%$ of clinics. In the remainder, it is measured twice or more per year in all but three clinics, where it is measured annually. Of clinics using HbAlc measurements, 86\% reported that the result was available during the clinic compared to $70 \%$ in 1998, 50\% using a "near-patient" methodology. 


\section{Complication monitoring}

- NICE Guideline 5.5: Children with type 1 diabetes should be offered screening for retinopathy, microalbuminuria, and blood pressure annually from the age of 12 years. ${ }^{5}$

Only $3 \%$ and $4 \%$ of clinics respectively stated that they never screen for microalbuminuria or retinopathy, with only one clinic never measuring blood pressure. Thirteen per cent of clinics screen for microalbuminuria and retinopathy at every visit, and 38\% check blood pressure at every visit. By age 12 years, $83 \%$ of clinics organise annual screening for microalbuminuria and $80 \%$ for retinopathy. These percentages are greater with early puberty or long duration of diabetes.

\section{Screening methods}

Methods used for microalbumin screening were: albumin/ creatinine ratio on early morning urine samples $(81 \%)$; timed overnight urine collection (14\%); and 24 hour urine collection $(5 \%)$. Methods for retinopathy screening include: nondilated fundoscopy (58\%); dilated fundoscopy by paediatrician $(25 \%)$ and by ophthalmologist $(38 \%)$; retinal photography $(51 \%)$.

\section{Screening for associated conditions}

- NICE Guideline 5.5: Children with type 1 diabetes should be offered screening for coeliac disease at diagnosis and at least every three years thereafter; thyroid disease at diagnosis and annually thereafter.

For coeliac disease 91 (69\%) test annually, six alternate years, four every 3 years, four once only, 19 if clinically indicated, and seven clinics ( $5 \%$ of responders) never test.

For thyroid disease $120(83 \%)$ test annually, seven alternate years, three every 3 years, 13 if clinically indicated, and one clinic never screens.

\section{Clinic registers and policies}

- Recommendation: There should be a district diabetes register and regular audit of outcomes. ${ }^{6}$

- NICE Guideline 5.3: All centres should have written protocols to ensure safe management of children with diabetic ketoacidosis and during surgery. ${ }^{5}$

Ninety five per cent of clinics have a register, which is electronic in three quarters. One hundred and nineteen clinics (75\% respondents) reported that they contribute data to the National Diabetes Audit run by Diabetes UK, ${ }^{8}$ although HbAlc data were only available in 2002 from $49 \%$.

One hundred per cent of clinics have a written protocol for diabetic ketoacidosis, $90 \%$ for surgery, and $74 \%$ for severe hypoglycaemia. Seventy one per cent have a written education curriculum for children and families.

\section{Transfer to adult services}

- Diabetes NSF Standard 6: All young people with diabetes should experience a smooth transition of care from paediatric diabetes to adult diabetes services. The transition should be organised in partnership and agreed with each individual. ${ }^{4}$

One hundred and fifteen respondents (71\% compared with $52 \%$ in 1998) have age stratified diabetes clinics, $63 \%$ of which are adolescent clinics, $35 \%$ young persons' clinics, and $69 \%(48 \%$ of total) organise joint clinics with adult colleagues. Eighty four per cent of the 125 respondents to this question reported that they had a policy or procedure for handover to adult care. This transfer takes place at 13-25 years of age (mean 16.7 years).

\section{Meeting the recommendations}

From the document "Principles of Good Practice for the Care of Young People with Diabetes", 10 key recommendations for a paediatric diabetes service were analysed and compared with the responses in $1998 .^{3}$

Improvements were noted in five recommendations: percentages of paediatricians with a special interest in diabetes, designated children's diabetes clinics, clinics of more than 40 patients, clinic attendance of DSNs, and microalbuminuria screening. There was no improvement in measurement of glycated haemoglobin. There was a decrease in availability of paediatric dieticians, frequency of retinopathy screening, and joint paediatric/adult hand-over clinics. In 2002, 61\% clinics had DSN:patient ratio of less than 1:100 (47\% in 1998); $97 \%$ met 5 of the 10 recommendations $(96 \%$ in 1998), $55 \%$ met 8 (58\% in 1998), and only $2 \%$ met all 10 recommendations (12\% in 1998).

\section{Clinics meeting the 10 key recommendations}

See table 2 .

\section{Relation with $\mathrm{HbAlc}$ level}

The mean clinic HbAlc levels (DCCT standardised) from the Diabetes UK/BSPED National Paediatric Diabetes Audit ${ }^{8}$ were compared with all the survey variables. Eighty three survey responders $(49 \%)$ had participated in the national audit and also provided HbAlc results. Two variables were found to have a significant relation with mean HbAlc level. Diabetes services provided by 10 general paediatricians were related to poorer HbAlc results (mean 9.4\%) than those provided by paediatricians with a special interest in diabetes, including paediatric diabetologists (mean 8.9\%; $p=0.002$ ). HbAlc levels were lower if the diabetes specialist nurses worked in both hospital and the community (mean $8.9 \%$ versus $9.3 \%$, $\mathrm{p}<0.05)$. Numbers of Specialist Nurses and dietitians were not directly related to HbAlc levels.

\section{DISCUSSION}

The results show improvements in several organisational aspects of paediatric diabetes care. Fewer consultants look after inappropriately small numbers of children and have significantly bigger caseloads. This has always been one aim of national recommendations - to centralise to some degree the expertise into larger clinics. Nevertheless there are still 12

Table 2 Percentage of respondents answering each question, meeting each of 10 key recommendations for paediatric diabetes care ${ }^{6}$

\begin{tabular}{llc}
\hline Recommendation & $\begin{array}{l}1998 \\
(\%)\end{array}$ & $\begin{array}{l}2002 \\
(\%)\end{array}$ \\
\hline Designated children's diabetes clinic & 91 & 98 \\
Paediatric diabetes specialist nurse & 75 & 98 \\
Clinic of more than 40 patients & 74 & 94 \\
Paediatrician with a special interest in diabetes & 78 & 88 \\
Glycated haemoglobin at each clinic visit & 88 & 86 \\
Microalbuminuria screening & 66 & $83^{*}$ \\
Retinopathy screening & 87 & $80^{*}$ \\
Paediatric dietician & 65 & 68 \\
Joint or combined hand-over clinic to adult & 78 & 48 \\
services & & \\
Paediatric diabetes specialist nurse:patient ratio & 53 & 39 \\
$<1: 100$ & & \\
No. of respondents meeting: $\dagger$ & 58 & 55 \\
8 recommendations & 29 & 33 \\
9 recommendations & 12 & 2 \\
10 recommendations &
\end{tabular}

*Percentage carrying out annual screening in children over age 12 years. †This analysis was only carried out in 2002 on those 80 clinics whose consultants replied to all of the questions. 


\section{What is already known on this topic}

- The care of children with diabetes should now meet national standards

- Three previous surveys have shown gradual improvements in services

paediatricians with no stated special diabetes interest who each look after more than 70 diabetic children.

The lack of a national list of specialists was one of the deficiencies noted in the 1998 survey which required attention. $^{3}$ Since 2000, Diabetes UK has maintained a list of consultant paediatricians looking after children with diabetes for the National Diabetes Audit, so we can now be more confident that we received replies from the majority of hospital clinics $(78 \%)$. This response is poorer than in previous surveys, and it is possible that the non-responders might represent a group of paediatricians where the services are even less satisfactory than those reported. The responding 187 consultants looked after a total of around 18500 children and young people (15 400 under 16 years), which is the majority of the estimated 20000 in the UK. ${ }^{9}$ Thus the survey should have provided representative indicators of care for those individuals throughout the UK.

For the first time, because of the linkage with the national diabetes audit, we have been able to show that the clinics of consultants with a special interest achieve significantly better HbAlc levels than those run by general paediatricians.

The publication and dissemination of the results of this and three previous surveys through professional groups and Diabetes UK may well have had an impact on service organisation leading directly to improvements. This model could equally be applicable to services for other chronic childhood disorders.

Unfortunately, there are continuing deficiencies and in some respects services appear to have deteriorated since the last survey. Although the percentage of paediatric DSNs attending clinics has improved, their workload has increased with a mean caseload of 147 patients per whole time equivalent nurse, compared with recommendations of between 70 and $100 .{ }^{7}$ Moreover, the percentage of paediatric dieticians attending clinics has fallen to $61 \%$ and many respondents commented that the exceptionally poor provision of specialised psychology/psychiatry services was the weakest aspect of their service, a problem which is highlighted by recommendations in the NICE Guidelines. These staffing figures cause great concern considering the continuing increase in incidence of diabetes, particularly in very young children whose families require a great deal of time, expertise, and emotional support, and the increasing complexity and sophistication of modern diabetes care. The new insulin regimens, including multiple injection therapy, require more detailed dietetic education including quantitation of carbohydrate intake. ${ }^{10}$ This is not possible when a WTE dietician has an average case-load of 830 patients.

The progressive improvements in the numbers of DSNs is gratifying, with nearly all clinics having a nurse available and more are reported to be children's nurse trained $(82 \%$ compared with $48 \%$ in 1998). There are now fewer working with adults (28\% compared with $42 \%$ ). These trends must represent changes in training, but national recommendations state that all children should be looked after by appropriately children's trained nurses. ${ }^{7}$ Paediatric DSNs commonly practise domiciliary education, so it was interesting to find that the ability to work in the community as well as hospital appears to have a beneficial effect in terms of HbAlc levels.

\section{What this study adds}

- Services for children have continued to improve over the last four years; children are seen in larger, more specialised clinics by consultants with an interest in diabetes

- However, only $2 \%$ of clinics surveyed met all 10 recommendations; in particular nursing caseload is larger than recommended

Both the NSF and NICE guidelines emphasise the benefits of joint working with adult colleagues in transition services. The finding that joint clinics appear to be less prevalent now than previously is worrying in view of the high nonattendance rates in young adult clinics when transitional arrangements are ineffective, and the associated high levels of serious vascular complications. ${ }^{11-14}$ Perhaps this finding reflects the fact that adult services are similarly overstretched and under-resourced. ${ }^{15}$

The last survey highlighted a lack of IT support. It is pleasing to report that $95 \%$ of clinics now hold a register of their diabetic children, and that $75 \%$ of these are held electronically. This must improve the ease with which local and national audits can be carried out, and so it remains disappointing that only around half of our respondents had been reporting to the Diabetes UK National Diabetes Audit. ${ }^{8}$

In conclusion, although there is increasing specialisation, with larger clinics being run by more specialist paediatricians, serious deficiencies remain. These deficiencies must be addressed if children and young people at the vulnerable transition stage are to be helped in preventing the significant rates of psychological and diabetes related complications currently being seen in the UK. ${ }^{12}{ }^{14} 16$ The serious lack of resourcing reported by many specialist teams caring for children with diabetes strongly support the contention that community care of this serious lifelong condition is not being recognised as a priority by purchasing authorities.

\section{Main conclusions}

This 4th National Survey confirms significant improvements in organisational structure including the concentration of expertise into bigger, more specialised clinics, but also highlights continuing important deficiencies including:

- Inadequate provision of appropriately trained and adequate numbers of specialised staff for children's diabetes teams, particularly children's trained diabetes specialist nurses and paediatric dieticians specialising in diabetes

- Particularly poor access to specialised psychology/psychiatry services

- Insufficient liaison and joint working with adult colleagues

- Variable standards in measuring glycated haemoglobin, and screening for complications or associated conditions.

\section{ACKNOWLEDGEMENTS}

We would like to thank Alex Smith, Lydia Jackson, and Sandra Kiauka of Diabetes UK who helped with the design and distribution of questionnaires, and collection of data.

\section{Authors' affiliations}

J A Edge, John Raddliffe Hospital, Oxford, UK

P G F Swift, Leicester Royal Infirmary Children's Hospital, Leicester, UK W Anderson, B Turner, Diabetes UK, London, UK

Competing interests: none declared 


\section{REFERENCES}

1 British Paediatric Association Working Party. The organisation of services for children with diabetes in the United Kingdom: report of the British Paediatric Association Working Party. Diabetic Med 1990;7:457-64.

2 Haines LC, Swift PGF. Report of the 1994 BPA/BDA survey of services for children with diabetes: changing patterns of care. Diabetic Med 1997; 14:693-7.

3 Jefferson IG, Swift PGF, Skinner TC, et al. Diabetes services in the UK: third national survey confirms continuing deficiencies. Arch Dis Child 2003;88:53-6.

4 NSF Diabetes. Standards. Clinical care of children and young people with diabetes. Health Service Circular (HSC 2001/026). Dept of Health, December 2001. www.doh.gov.uk/nsf/diabetes.

5 CG15. Diagnosis and management of type 1 diabetes in children and young people. National Institute for Clinical Excellence, July 2004. www.nice.org.uk.

6 Anon. The principles of good practice for the care of young people with diabetes. A British Diabetic Association Report. London, 1995.

7 Anon. The role and qualifications of the nurse specialising in paediatric diabetes. London: Royal College of Nursing of the United Kingdom, Paediatric Diabetes Special Interest Group, 1993.
8 Smith A, Jefferson I. The National Paediatric Diabetes Register/Audit. Diabetic Med 2001; 18:409-12.

9 Edge JA, Ford-Adams ME, Dunger DB. Causes of death in children with type 1 diabetes, 1990-1996. Arch Dis Child 1999;81:318-23.

10 Tamborlane WV, Bonfig W, Boland E. Recent advances in treatment of youth with type 1 diabetes: better care through technology. Diabetic Med 2001; 18:864-70.

11 Betts PR, Jefferson IG, Swift PG. Diabetes care in childhood and adolescence. Diabetic Med 2002; 19(suppl 4):61-5.

12 Bryden KS, Dunger DB, Mayou RA, et al. Poor prognosis of young adults with type 1 diabetes: a longitudinal study. Diabetes Care 2003;26:1052-7.

13 Kipps S, Bahu T, Ong K, et al. Current methods of transfer of young people with type 1 diabetes to adult services. Diabetic Med 2002;19:649-54.

14 Roberts SE, Goldacre MJ, Neil HAW. Mortality in young people admitted to hospital for diabetes: database study. BMJ 2004;328:741-2.

15 Parr JH, Kelly WF, Large DM, et al. Improvements in the provision of hospital diabetes services in an English region 1988-98. Diabetic Med 2002; 19/suppl 4):79-82.

16 Scott A, Donnelly R. Improving outcomes for young people with diabetes. Diabetic Med 2001;11:861-3.

\section{1 th European Forum on Quality Improvement in Health Care}

26-28 April 2006, Prague, Czech Republic For further information please go to: www.quality.bmipg.com Book early to benefit from a discounted delegate rate 